\title{
MOTIVAÇÃO PARA APRENDER: DIFERENÇAS DE METAS DE REALIZAÇÃO ENTRE ALUNOS DO ENSINO FUNDAMENTAL
}

\author{
Rebecca de Magalhães Monteiro \\ Mestre e doutoranda em Psicologia no Programa de Pós-Graduação Stricto Sensu da Universidade \\ São Francisco. \\ Acácia Aparecida Angeli dos Santos \\ Doutora em Psicologia pelo Instituto e Psicologia da USP. Docente da graduação e da Pós- \\ graduação Stricto Sensu da Universidade São Francisco. Bolsista Produtividade do CNPq.
}

\begin{abstract}
Resumo
O presente trabalho investigou as metas de realização de alunos do $3^{\circ}$ ao $5^{\circ}$ ano do Ensino Fundamental de uma escola pública e outra particular de Belo Horizonte. Também foram exploradas as diferenças relativas às variáveis tipo de escola e ano escolar. Participaram 518 crianças com idades entre 7 e 13 anos. O instrumento utilizado foi a Escala de Avaliação da Motivação para a Aprendizagem - EMAPRE-I composta pelos fatores meta aprender, meta performance-aproximação e meta performance-evitação. Os resultados revelaram que a meta predominante entre os alunos dessa amostra foi a meta performance-evitação e que houve uma diferença estatisticamente significativa entre as médias das metas quanto a variável tipo de escola, favorecendo os alunos da escola pública. Em relação ao ano escolar as médias das metas aprender e performance-evitação apresentaram diferenças significativas entre os anos escolares da escola particular, favorecendo os alunos do $3^{\circ}$ ano e os do 50 ano, respectivamente.
\end{abstract}

Palavras-chave: motivação, ensino fundamental, avaliação psicoeducacional.

\section{LEARNING MOTIVATION: DIFFERENCES BETWEEN THE ACHIEVEMENT GOALS OF THE ELEMENTARY SCHOOL STUDENTS}

\begin{abstract}
This study investigated the achievement goals of students from 3rd to 5th year of elementary school from a public and a private school of Belo Horizonte. Even explored differences related to the variables as school type and school year. The participants consisted of 518 children with ages ranging from 7 to 13 years old. The instrument used was the Escala de Avaliação da Motivação para a Aprendizagem - EMAPRE-I that consists of three factors, learning goal, performance-approach goal and performanceavoidance goal. The results revealed that the most prevalent goal among students in this sample was the performance-avoidance goal and there was a statistically significant difference between goals means of the variable type of school, favouring public school students. In relation to the average goals of school year, learning goal and performance-avoidance goal showed significant differences between the school years of the private school, favouring students year 3 and year 5 , respectively. Key-words: motivation, elementary school, psychoeducational assessment.
\end{abstract}




\title{
MOTIVACIÓN PARA APRENDER: DIFERENCIAS ENTRE LAS METAS DE REALIZACIÓN DE LOS ESTUDIANTES DE LA ESCUELA PRIMARIA
}

\begin{abstract}
Resumen
El presente trabajo investigó las metas de realización de estudiantes de $3^{\circ} .5^{\circ}$ año de enseñanza básica de dos escuelas de Belo Horizonte: una pública y otra privada. También exploró diferencias relativas a las variables: tipo de escuela y año escolar. Participaron 518 niños y niñas con edades entre 7 y 13 años. El instrumento utilizado fue la Escala de Avaliação da Motivação para a Aprendizagem - EMAPRE-I. Esta escala es compuesta por los factores: meta-aprender; meta-performance-aproximación y meta-performance-aproximación. Los resultados revelaron que la meta predominante entre los estudiantes de la muestra, fue la meta-performance-evitación y que hubo una diferencia estadísticamente significativa entre las medias de las metas en cuanto a la variable tipo de escuela, favoreciendo los estudiantes de la escuela pública. En relación al año escolar, las medias de las metas aprender y performance-evitación presentaron diferencia significativa entre los años escolares en la escuela privada, favoreciendo los estudiantes del $3^{\circ}$ año y del $5^{\circ}$ año respectivamente.
\end{abstract}

Palabras-clave: motivación, escuela primaria, evaluación psico-educacional.

\section{INTRODUÇÃO}

A motivação é considerada uma variável-chave para a aprendizagem, já que o empenho dos alunos em aprender e as estratégias adotadas com tal propósito são fundamentais no ambiente da escola. Alguns estudos, realizados na última década, apontam a importância desse construto no contexto escolar da criança devido a sua relação com o desempenho acadêmico (Givvin, 2001; Gouveia, Diniz, Gouveia \& Cavalcante, 2008; Martinelli \& Genari, 2009; entre outros).

É possível dizer que as atividades acadêmicas apresentam mais de um significado e que contribuem para a consecução de diferentes metas. Nesse sentido, nem todas as metas têm o mesmo significado para cada aluno, visto que suas interpretações podem variar segundo as orientações pessoais e as situações acadêmicas que os estudantes enfrentam ao longo da vida (Ames, 1992; Tapia \& Montero, 2004; Shih, 2005; entre outros). Dessa forma, Zenorini (2007) destaca que a motivação no ambiente escolar é vista como um dos principais fatores que favorecem a aprendizagem dos alunos. Um estudante motivado, possivelmente, demonstra maior envolvimento e esforço no processo de aprendizagem, enfrenta tarefas desafiadoras, não desanima diante do insucesso, persiste ao realizar as tarefas e utiliza estratégias mais adequadas.

Uma das teorias que busca a compreensão dos fatores motivacionais que podem influenciar o comportamento do aluno especificamente atinente às situações de aprendizagem escolar é a Teoria de Metas de Realização surgida no 
final dos anos 70 do século passado (Dweck, 1986). Essa teoria, de acordo com Zenorini (2007), caracteriza o termo meta como sendo o aspecto qualitativo do envolvimento do aluno com situações de aprendizagem, expressando em nível hierárquico o propósito de uma pessoa dedicar-se a uma tarefa. Desse modo, a meta de realização que a pessoa adota representa o motivo ou a razão pela qual ela irá realizar determinada tarefa. As metas de realização podem ser consideradas como um conjunto de pensamentos, crenças, propósitos e emoções que traduzem as expectativas dos alunos em relação a determinadas tarefas que deverão executar (Ames, 1992).

Em geral, os professores sabem que os alunos investem seus esforços acadêmicos com diferentes metas. Existem os alunos que buscam tirar uma nota alta, os que passam de ano com o mínimo de dedicação necessária, os que se comparam o tempo todo com os colegas e querem obter a melhor nota da sala e os que querem aprender, simplesmente, para adquirir mais conhecimento. De acordo com a Teoria de Metas de Realização, essas metas podem facilitar as experiências dos alunos durante o período de escolarização (Bzuneck, 2004b; Senko \& Harackiewicz, 2005; Tapia \& Montero, 2004; entre outros).

Os estudos iniciais dessa teoria identificaram três metas com características distintas, a meta aprender, a meta performance e alienação acadêmica. Para Dweck e Leggett (1988), o aluno voltado à meta aprender tem a convicção de que os resultados positivos nas tarefas derivam de esforço, que é um fator interno. Este objetivo corresponde ao desejo de aprender e adquirir conhecimento. Diversos estudos têm demonstrado que a meta aprender pode levar a ações adaptativas, tais como esforço e persistência após o fracasso (Darnon, Harackiewicz, Butera, Mugny \& Quiamzade, 2007; Zenorini, Santos \& Bueno, 2003; Zenorini \& Santos, 2010). Nesse contexto, os alunos acreditam que o sucesso escolar é alcançado mediante o esforço, a dedicação e o aprendizado adquirido naquela tarefa.

Para o aluno voltado à meta performance, os resultados são associados ao nível de capacidade, ou seja essa meta destina-se mais a uma orientação externa de pressões e exigências sociais. A preocupação do sujeito volta-se para a opinião do outro em relação às suas conquistas, sendo o reconhecimento considerado fundamental (Elliot \& Church, 1997). Segundo Bzuneck (2004b), a meta performance, também chamada de capacidade ou de ego, pode ser dividida em dois aspectos, a saber, a meta performance-aproximação e meta 
performance-evitação. A primeira caracteriza-se pela busca de parecer inteligente, mostrar-se capaz e até mesmo querer ser o melhor em comparação com seus pares. A segunda trata do medo do fracasso, sendo que o sujeito orientado pela meta performance-evitação demonstra uma preocupação em não parecer incapaz diante dos outros colegas. Portanto, mostrar-se capaz ou não capaz é a grande questão do aluno caracterizado pela meta performance (Ee, Wang, Koh, Tan \& Liu, 2009).

Algumas pesquisas apontam que a meta performance-aproximação correlaciona-se positivamente com a meta aprender e o desempenho acadêmico, ou seja essa meta nem sempre tem sido negativa no ambiente escolar (Bzuneck, 2004b; Gouveia \& cols., 2008; Shih 2005; entre outros). Já no estudo de Middleton e Midgley (1997) a meta performance-aproximação apareceu relacionada positivamente a comportamentos de evitação em sala de aula e também à ansiedade. Ao contrário da meta performance-aproximação, existe um consenso entre as pesquisas sobre a meta performance-evitação, pois ela tem apresentado correlação positiva com a ansiedade, e negativa com a autoeficácia, com estratégias cognitivas e com o desempenho acadêmico (Boruchovitch \& Costa, 2004; Gouveia \& cols., 2008; Middleton \& Midgley, 1997; Shih 2005; entre outros).

A meta alienação acadêmica ou de evitação do trabalho, diz respeito à falta de preocupação do aluno em aumentar sua competência ou demonstrar capacidade, mas sim de executar as tarefas escolares com o mínimo de esforço. O resultado da tarefa não tem importância para o aluno que possui a meta alienação acadêmica, possivelmente porque sua auto-estima pode ser garantida em atividades fora da escola (Bzuneck 2004b). Além disso, o que mais importa para o sujeito é ficar livre das obrigações escolares e escolher aquilo que exige menos esforço. Embora essa meta não seja abordada na escala de motivação escolhida para o presente estudo, considerou-se importante citá-la, visto que ela também é encontrada em estudos que adotam a Teoria de Metas de Realização.

Percebe-se que a motivação do aluno no contexto escolar é associada a Metas de Realização, que correspondem a um conjunto de cognições ou esquemas mentais envolvendo propósitos, crenças, atribuições e percepções que podem levar a decisões comportamentais e a reações afetivas. O aspecto da teoria de metas que a torna particularmente relevante à educação é que ela explica a qualidade do envolvimento do aluno nas atividades escolares (Ames, 
1992; Bzuneck, 2004b; Elliot \& Church, 1997; Gouveia e cols., 2008; Senko \& Harackiewicz, 2005; Tapia \& Montero, 2004; Zenorini, 2007).

Ao analisar as experiências de aprendizagem nas escolas, Gottfried (1990) aponta que com o passar dos anos de escolarização as pressões e exigências referentes ao desempenho desejável aumentam e, consequentemente, os alunos orientam-se por uma motivação mais extrínseca. Isso significa que no decorrer da sua entrada na escola, o envolvimento do aluno abre espaço para questões externas, como notas, avaliações, reconhecimento e valorização pelo trabalho desenvolvido (Broussard \& Garrison, 2004; Bzuneck 2004a; Guimarães, 2004; entre outros).

Sob essa perspectiva Broussard e Garrison (2004) acreditam que é essencial avaliar o processo de motivação em crianças dos anos iniciais do ensino fundamental. A motivação orientada por questões mais intrínsecas ao sujeito, na entrada da escola, pode contribuir para o sucesso futuro desses alunos. Para os autores, o desenvolvimento de crianças voltadas para a meta aprender é, particularmente, importante por representar crenças, valores e práticas estabelecidas logo nos primeiros anos da escola e que, possivelmente, farão parte dos seus comportamentos futuros. Padrões motivacionais em crianças mais velhas estão associados a padrões adquiridos anteriormente, tal como apontado nos estudos que se seguem, realizados com alunos do ensino fundamental.

O estudo longitudinal de Anderman e Anderman (1999) objetivou investigar as relações entre a percepção social e as orientações de metas de 660 estudantes de 21 escolas de ensino fundamental. Nesse estudo as metas foram divididas em meta aprender e meta performance, não tendo sido diferenciada a meta performance em aproximação e evitação. A pesquisa ocorreu, quando os alunos faziam a transição da $5^{a}$ série para a $6 a$ série.

Os resultados apresentados por Anderman e Anderman (1999) indicaram que os estudantes da $6^{a}$ série relataram níveis mais baixos na meta aprender do que na $5^{a}$ série. Por outro lado os estudantes da $6^{a}$ relataram níveis mais altos na meta performance do que na $5^{a}$ série. A meta aprender na $6^{a}$ série correlacionou-se positivamente com a percepção dos estudantes com o senso de participação escolar na escola e com a meta de responsabilidade social, também se correlacionou significativamente, embora modestamente, com a meta de relações sociais. Já a meta performance correlacionou-se significativamente e de forma positiva com a meta status social e modestamente com a meta de 
relações sociais. Com relação ao desempenho desses estudantes, a correlação das notas mais altas e a meta performance foi negativa.

O estudo de Broussard e Garrison (2004) realizado com 249 crianças do primeiro e do terceiro ano do Ensino Fundamental, provenientes de 17 escolas localizadas no sul dos Estados Unidos, teve como objetivo verificar a relação existente entre as orientações motivacionais e o desempenho em matemática e leitura. Nesse estudo os autores utilizaram os conceitos de motivação intrínseca, que se refere à disposição natural e espontânea que impulsiona a pessoa a buscar novidades e desafios, e motivação extrínseca apontada como a motivação para trabalhar em resposta a algo externo à atividade para obter recompensas e reconhecimentos.

Os resultados apontaram uma correlação positiva e significativa entre a motivação intrínseca e o desempenho em matemática nos alunos do primeiro ano. Já em relação aos alunos do terceiro ano, o desempenho em matemática e leitura correlacionaram positiva e significativamente com a motivação intrínseca e extrínseca. Por meio da análise de regressão foi identificado que a motivação intrínseca pode predizer o desempenho de crianças no primeiro ano, enquanto as duas motivações, intrínseca e extrínseca, são preditoras do desempenho de estudantes do terceiro ano.

Os achados da pesquisa de Broussard e Garrison (2004) indicaram que crianças mais novas e que estão no início da escolarização, orientam-se mais pela motivação intrínseca quando comparadas com os alunos que estão em séries mais avançadas. Os autores acreditam que um dos aspectos explicativos dessa diferença é o fato de as crianças mais velhas já perceberem e se preocuparem com as críticas, cobranças e exigências dos pais e da própria escola.

Conforme discutido nos estudos de Anderman e Anderman (1999) e Broussard e Garrison (2004) percebe-se a importância de se estudar a motivação nos anos iniciais do Ensino Fundamental. Com este propósito o presente estudo buscou investigar as Metas de Realização de alunos do terceiro ao quinto ano do Ensino Fundamental de uma escola pública e outra particular de Belo Horizonte. Também foram objetivos desta pesquisa explorar eventuais diferenças relativas às variáveis tipo de escola e ano escolar. 


\section{MÉTODO}

\section{Participantes}

Participaram da pesquisa 518 alunos devidamente matriculados do $3^{\circ}$ ao $5^{\circ}$ ano do Ensino Fundamental provenientes de uma escola particular e uma escola pública de Belo Horizonte, sendo 54,2\% $(n=281)$ do sexo masculino e 45,8\% $(n=237)$ do sexo feminino. Os participantes da escola particular foram 363 alunos, $32 \%(n=116)$ do 30 ano, 33\% $(n=120)$ do 40 ano e $35 \%(n=127)$ do 50 ano. Quanto ao sexo 56,2\% $(n=204)$ eram meninos e 43,8\% $(n=159)$ eram meninas. Em relação aos 155 participantes da escola pública, 36,8\% $(n=57)$ eram do 30 ano, 32,3\% $(n=50)$ do 40 ano e $31 \%(n=48)$ do 50 ano, os meninos totalizaram $49,7 \%(n=77)$ dessa amostra e as meninas $50,3 \%(n=78)$.

A faixa etária dos alunos, na amostra total, variou de 7 a 13 anos de idade $(M=9,10 ; D P=1,205)$. Na escola particular a idade mínima foi de 7 anos e a máxima 11 anos $(M=8,85 ; D P=0,91)$, enquanto na escola pública as idades variaram entre 7 e 13 anos $(M=9,69 ; \quad D P=1,56)$. A amostra foi nãoprobabilística, já que a escolha foi feita conforme a conveniência e facilidade de contato com as escolas.

\section{Instrumento}

Escala de Avaliação da Motivação para a Aprendizagem - versão infantil EMAPRE-I (Zenorini \& Santos, 2009)

A escala versão infantil utilizada no presente estudo é composta pelos fatores, meta aprender (11 itens), meta performance-aproximação (11 itens) e meta performance-evitação (3 itens), totalizando 25 itens. As opções de respostas apresentadas para os alunos classificam-se em $1=$ sempre, $2=$ às vezes e 3=nunca. A pontuação máxima é de 75 e mínima de 25 pontos. Em relação aos estudos psicométricos a escala apresentou evidência de validade por meio da estrutura interna, já que a análise fatorial exploratória indicou a existência de três fatores, correspondentes às três subescalas previstas teoricamente e os itens obtiveram carga fatorial acima de 0,40. Quanto à consistência interna o alfa de Cronbach por dimensão foi de 0,77 para a meta aprender, 0,86 para meta performance-aproximação e 0,61 para a meta performance-evitação, a escala total apresentou o valor de 0,80. Para as análises estatísticas do presente trabalho a média de cada meta foi retirada de acordo com a média ponderada, 
ou seja, o valor bruto de cada meta foi dividido pelo número de itens de cada uma e multiplicado por 10.

\section{Procedimento}

Primeiramente foi solicitada a autorização da direção das escolas para a realização da pesquisa e, em seguida, o projeto foi encaminhado para a aprovação do Comitê de Ética. Os participantes foram informados sobre os objetivos do estudo e sobre a exigência da assinatura do Termo de Consentimento Livre e Esclarecido (TCLE) pelo responsável, já que os alunos eram menores de idade.

As informações sobre a pesquisa foram transmitidas para as crianças e os pais participantes, inicialmente, pelas diretoras das escolas e, posteriormente, no dia da aplicação dos testes pelas pesquisadoras. Para os pais das crianças foi feito um comunicado por escrito, por meio de uma circular das escolas e junto foi encaminhado o TCLE para os pais autorizarem ou não a participação de seus filhos na pesquisa. Em seguida a EMAPRE-I foi aplicada coletivamente nos alunos em sala de aula, durante o período de aula, no horário liberado pela direção das escolas. O tempo gasto para a realização dessa tarefa foi de 30 minutos.

\section{RESULTADOS}

Após a aplicação e correção do instrumento os dados foram analisados quantitativamente utilizando-se provas de estatística descritiva e inferencial. A primeira possibilitou a descrição do desempenho dos participantes no instrumento utilizado. As provas inferenciais forneceram evidências sobre as diferenças de médias relativas às variáveis tipo de escola e ano escolar.

Tabela 1.

Estatísticas descritivas das médias dos sujeitos em relação às metas de realização.

\begin{tabular}{cccccc}
\hline Metas de Realização & $\mathrm{n}$ & Mínimo & Máximo & $\mathrm{M}$ & $\mathrm{DP}$ \\
\hline Meta aprender & 477 & 1,36 & 3,00 & 2,52 & 0,32 \\
Meta performance-aproximação & 475 & 0,82 & 2,45 & 1,57 & 0,40 \\
Meta performance-evitação & 489 & 2,33 & 7,00 & 3,83 & 1,25 \\
\hline
\end{tabular}

A primeira parte das análises realizadas consistiu em avaliar a média dos alunos em relação às Metas de Realização avaliadas pela EMAPRE-I. Os 
resultados das provas de estatística descritiva são apresentados nas Tabelas 1 e 2.

Observa-se que na amostra total os alunos obtiveram uma maior média na meta performance-evitação $(M=7,00 ; D P=1,25)$. Ressalta-se que foi calculada a média ponderada de cada meta avaliada pela EMAPRE - I, ou seja, o total de cada uma foi dividido pelo respectivo número de itens. Dessa maneira, pôde-se obter o resultado de predominância de meta, já que elas foram comparadas de maneira semelhante.

Tabela 2.

Estatísticas descritivas das médias dos sujeitos em relação às metas de realização separados por ano escolar.

\begin{tabular}{ccccc}
\hline Ano & $\mathrm{n}$ & Meta Aprender & $\begin{array}{c}\text { Meta performance- } \\
\text { aproximação }\end{array}$ & $\begin{array}{c}\text { Meta performance- } \\
\text { evitação }\end{array}$ \\
\hline $3^{\circ}$ ano & 143 & 2,59 & 1,58 & 3,65 \\
$4^{\circ}$ ano & 146 & 2,50 & 1,59 & 3,92 \\
$5^{\circ}$ ano & 156 & 2,48 & 1,53 & 3,94 \\
\hline
\end{tabular}

Verifica-se na Tabela 2 que as médias da meta aprender diminuem conforme o avanço dos alunos nos anos escolares, enquanto que as médias da meta performance-evitação aumentam. Além disso, em todos os anos estudados existe uma predominância da meta performance-evitação conforme também apontado na Tabela 1.

A fim de explorar eventuais diferenças associadas ao tipo de escola e ano escolar foram utilizadas análises de variância, teste t de Student e ANOVA. Os resultados obtidos estão descritos nas próximas tabelas.

Tabela 3.

Estatísticas descritivas e Teste $\mathrm{t}$ de Student para comparação das médias ponderadas das Metas de Realização em relação ao tipo de escola.

\begin{tabular}{ccccccc}
\hline Metas & Escola & $\mathrm{n}$ & $\mathrm{M}$ & $\mathrm{DP}$ & $\mathrm{t}$ & $\mathrm{p}$ \\
\hline \multirow{2}{*}{ Meta aprender } & Particular & 342 & 2,49 & 0,32 & $-3,821$ & 0,001 \\
& Pública & 135 & 2,61 & 0,31 & & \\
Meta performance- & Particular & 345 & 1,45 & 0,34 & $-11,229$ & 0,001 \\
aproximação & Pública & 130 & 1,88 & 0,38 & & \\
Meta performance- & Particular & 351 & 3,59 & 1,07 & $-6,378$ & 0,001 \\
evitação & Pública & 138 & 4,46 & 1,44 & & \\
\hline
\end{tabular}


Verifica-se que as médias dos alunos da escola pública foram superiores aos dos alunos da escola particular na meta aprender, meta performanceaproximação e meta performance-evitação. As diferenças entre as médias, por tipo de instituição, apresentaram valores estatisticamente significativos $(p<0,001)$ para os três tipos de metas, sendo a diferença mais acentuada a relativa à meta performance-aproximação.

A próxima análise explorou as diferenças entre as médias das metas de realização entre os anos estudados utilizando-se a ANOVA. O resultado dessa análise indicou diferença estatisticamente significativa somente na meta aprender $[F(2,474)=5,092 ; p<0,006]$ em relação aos anos escolares. Para verificar como os anos se diferenciaram, utilizou-se o teste post-hoc de Tukey.

Tabela 4.

Agrupamento dos anos em relação aos escores da meta aprender no teste de Tukey.

\begin{tabular}{cccc}
\hline Ano & N & \multicolumn{2}{c}{ Médias da Meta Aprender } \\
& & 1 & 2 \\
\hline 30 ano & 163 & 2,59 & \\
40 ano & 157 & & 2,50 \\
50 ano & 157 & & 2,48 \\
\hline
\end{tabular}

O teste de Tukey separou os três anos escolares em dois agrupamentos, constatando a existência de uma diferença significativa do terceiro ano para o quarto e o quinto ano. A maior média na meta aprender foi a do terceiro ano que se diferenciou significativamente das obtidas pelos alunos do quarto ano e do quinto ano.

Também foi realizada análise de variância entre os escores das metas de realização por ano escolar separados por tipo de escola. Os resultados revelaram existir uma diferença estatisticamente significativa em relação às médias dos anos na meta aprender $[F(2,339)=4,178 ; p<0,016]$ e na meta performanceevitação $[F(2,348)=6,353 ; p<0,002]$ na escola particular. Na escola pública não foram detectadas diferenças estatisticamente significativas na comparação entre os anos. Os resultados do teste post-hoc de Tukey utilizado para identificar como ocorreram as diferenças na escola particular relativas à meta aprender e performance-evitação encontram-se na Tabela 5. 
Tabela 5.

Agrupamento das séries em relação aos escores da meta aprender e meta performance-evitação na escola particular.

\begin{tabular}{cccc}
\hline & & \multicolumn{2}{c}{ Médias da Meta Aprender } \\
Ano & & 1 & 2 \\
\hline $3^{\circ}$ ano & 112 & 2,56 & 2,46 \\
40 ano & 112 & 2,46 & 2,44 \\
\hline 50 ano & 118 & Médias da Meta Performance-evitação \\
\hline & & 1 & 2 \\
\hline 30 ano & 112 & 3,30 & 3,67 \\
40 ano & 118 & & 3,78 \\
\hline 5
\end{tabular}

O teste post-hoc de Tukey mostrou uma diferença estatisticamente significativa entre o terceiro e o quinto ano, sendo a maior média obtida pelo terceiro ano. A média dos alunos do quarto ano não se diferenciaram significativamente das demais. Quanto ao resultado das médias da meta performance-evitação, o terceiro ano se diferenciou estatisticamente do quarto e quinto ano. Neste caso o terceiro ano obteve a menor média em relação aos demais.

\section{DISCUSSÃO DOS RESULTADOS}

A motivação para a aprendizagem pode ser considerada como um dos determinantes da qualidade do envolvimento do aluno com as atividades escolares (Ames, 1992; Bzuneck, 2004b; Elliot \& Church, 1997; Senko \& Harackiewicz, 2005; Zenorini, 2007). No ambiente de sala de aula as tarefas propostas pelos professores podem variar de acordo com o propósito da disciplina e de seus objetivos. Dessa forma, nem todas as tarefas têm o mesmo significado para cada aluno, visto que suas interpretações podem se diferenciar segundo as orientações pessoais, as situações acadêmicas que os estudantes enfrentam ao longo da vida e as metas para realizar tais tarefas (Ames, 1992; Tapia \& Montero, 2004; Shih, 2005; entre outros). A meta utilizada pelos alunos para a realização das atividades escolares foi um dos aspectos que norteou a exploração das características motivacionais dos estudantes aqui pesquisados. 
Com relação às metas de realização identificadas observou-se uma predominância da meta performance-evitação na amostra total pesquisada. Uma das implicações da meta performance-evitação é o aumento da ansiedade frente a provas e testes, assim como a inibição do aluno na hora de fazer perguntas em sala de aula, por exemplo (Boruchovitch \& Costa, 2004). Isso sugere, que os participantes do presente estudo tendem a se preocupar de uma maneira negativa com as exigências e pressões do ambiente acadêmico.

A segunda meta com maior média, porém bem inferior à meta anterior, foi a meta aprender que é identificada como a orientação motivacional ideal pelo fato do aluno trabalhar em função de adquirir competência e conhecimento por meio de esforço e persistência, sem a preocupação expressiva com a nota ou recompensas (Darnon \& cols., 2007; Zenorini \& cols., 2003). Aspectos positivos da meta aprender também apareceram no estudo de Anderman e Anderman (1999) pois ela correlacionou-se de forma significativa com o senso de participação escolar e com a meta de responsabilidade social. Dados os resultados aqui obtidos, percebe-se que os participantes desta pesquisa não se esforçam tanto para adquirir mais conhecimento e sim para obter a nota final, ou seja preocupam-se mais com a performance.

Finalmente, a meta com menor prevalência foi a meta performanceaproximação também voltada para a demonstração de capacidade e preocupação com um reconhecimento externo. Algumas pesquisas apontam correlações positivas dessa meta com o desempenho acadêmico, com a meta aprender e com estratégias de aprendizagem (Gouveia \& cols., 2008; Shih, 2005; entre outros). Isso ocorre devido ao propósito do aluno de alcançar o melhor resultado possível. No entanto, a literatura apresenta resultados contraditórios com relação a essa meta, como na pesquisa de Middleton e Midgley (1997) em que ela apareceu correlacionada positivamente a comportamentos de evitação em sala de aula e também à ansiedade. Essa é uma meta que gera mais discussão entre os estudiosos e que foi pouco revelada entre os alunos dessa amostra. Porém, embora exista uma diferença entre as metas performance-aproximação e performance-evitação, pode-se dizer que as duas se assemelham no que diz respeito à preocupação de exigências sociais e recompensas. Dessa maneira, o predomínio de uma dessas metas pode sugerir também um clima de competição entre os alunos. 
Conforme ressaltado por Broussard e Garrison (2004) é importante avaliar o processo de motivação em crianças dos anos iniciais do ensino fundamental, já que a orientação motivacional na entrada da escola pode contribuir para o sucesso futuro desses alunos. Em acréscimo os autores afirmam que o desenvolvimento de crianças voltadas para a meta aprender é fundamental por representar valores e práticas estabelecidas logo nos primeiros anos da escola e que, possivelmente, farão parte dos seus comportamentos futuros. Sendo assim, verificou-se neste estudo a possibilidade da meta aprender prevalecer nos anos iniciais do ensino fundamental. Os resultados a seguir discutem esses achados no presente trabalho.

Em relação às análises de variância das Metas de Realização constatou-se nesta amostra que os alunos do terceiro ano obtiveram uma maior média na meta aprender, seguido do quarto ano e por fim do quinto ano. Assim, verificouse uma diferença estatisticamente significativa na meta aprender em relação aos anos escolares. Estes achados corroboram com os estudos de Anderman e Anderman (1999) e Broussard e Garrison (2004), ao revelarem que os alunos tendem a se orientarem mais pela meta aprender em anos escolares iniciais. Outro dado que corrobora essa afirmação é o resultado da análise de variância por ano na escola particular. O resultado da ANOVA revelou existir uma diferença estatisticamente significativa em relação às médias dos anos escolares na meta performance-evitação também, visto que os alunos do quinto ano apresentaram média superior na meta performance-evitação, seguidos dos alunos do quarto ano e por fim dos do terceiro ano.

Na concepção de Bzuneck (2004a) e Gottfried (1990) esse fenômeno pode ser explicado pelo avanço na escolarização que muitas vezes contribui para uma maior conscientização das exigências e cobranças de notas e de resultados sempre melhores. Há que se mencionar também que para Guimarães (2004) a motivação voltada para a aprendizagem pode ser vista como um estado e uma disposição do sujeito aliados aos seus componentes cognitivos e afetivos. Nesse sentido, é possível inferir que as crianças menores estão mais voltadas para a curiosidade, o interesse e o desejo de conhecer coisas novas. Após sua entrada na escola, esse tipo de envolvimento abre espaço para questões externas ao aluno, como notas, avaliações, reconhecimento e valorização pelo trabalho desenvolvido. Dessa maneira, os resultados desta pesquisa revelaram essa 
tendência dos alunos mais velhos para a meta performance-aproximação e mais especificamente para a meta performance-evitação.

Vale salientar que a motivação do aluno no contexto escolar é associada a metas de realização, que correspondem a um conjunto de cognições ou esquemas mentais envolvendo propósitos, crenças, atribuições e percepções que podem levar a decisões comportamentais e a reações afetivas (Ames, 1992; Bzuneck, 2004b; Elliot \& Church, 1997; Gouveia \& cols, 2008; Senko \& Harackiewicz, 2005; Tapia \& Montero, 2004; Zenorini \& Santos, 2010). No entanto, muitas vezes as decisões comportamentais e reações afetivas estão relacionadas às atitudes e métodos adotados pela escola e pelo professor em sala de aula. Portanto, é possível dizer que as orientações motivacionais encontradas neste estudo também refletem as experiências vivenciadas pelos alunos no ambiente escolar.

\section{CONSIDERAÇÕES FINAIS}

Sabe-se que as exigências e as pressões no ambiente escolar podem direcionar mais ou menos para um clima competitivo entre os alunos, portanto é possível dizer que o professor e a escola são determinantes no desenvolvimento das metas de realização. Atitudes em sala de aula que estimulam a comparação entre os pares e a valorização do melhor resultado, por exemplo, acabam dando um significado de que a aprendizagem só pode ser medida por nota ou conceitos.

Ao considerar a predominância da meta performance-evitação na amostra desta pesquisa, pode-se pensar que alunos desde os anos iniciais da escola preocupam-se mais com a performance do que com a aquisição do conhecimento, das habilidades e competências que ocorrem durante o processo educativo. Vale destacar que essa meta caracteriza-se também pelo medo do fracasso, inibindo o aluno de se expor no ambiente de sala de aula.

A partir dos resultados encontrados, surgem algumas questões a fim de refletir sobre uma prática promotora de um bom envolvimento do aluno em relação às atividades escolares. Como intervir para que a meta aprender e/ou a meta performance-aproximação possam ser predominantes? Isso se forem considerados os aspectos positivos da meta performance-aproximação com o desempenho acadêmico. Como evitar que com o avanço da escolarização a meta performance-evitação se acentue? 
Vale destacar também que em um contexto onde a competitividade é grande ou incentivada, o tipo de orientação motivacional pode relacionar-se mais às questões externas, como notas, reconhecimento e valorização pelo outro. Por isso torna-se fundamental a reflexão sobre as práticas educacionais independente do tipo de escola, já que no presente estudo a escola particular e a escola pública apresentaram a mesma predominância de metas de realização.

\section{REFERÊNCIAS}

Ames, C. (1992). Classrooms: Goals, structures and student motivation. Journal of Educational Psychology, 84(3), 261-271.

Anderman, H. L., \& Anderman, E. M. (1999). Social predictors of changes in students' achievement goal orientations. Contemporary Educational Psychology, 25, 21-37.

Boruchovitch, E. \& Costa, E. R. (2004). O impacto da ansiedade no rendimento escolar e na motivação de alunos. In E. Boruchovitch \& J. A. Bzuneck (Orgs.), A motivação do aluno: contribuições da psicologia contemporânea. (3a ed., pp.134-147). Petrópolis, RJ: Vozes.

Broussard, S. C., \& Garrison, M. E. B. (2004). The relationship between classroom motivation and academic achievement in elementary-school-aged children. Family and Consumer Sciences Research Journal, 33, 106-120.

Bzuneck, J. A. (2004a). A orientação do aluno: aspectos introdutórios. In E. Boruchovitch \& J. A. Bzuneck (Orgs.), A motivação do aluno: Contribuições da Psicologia Contemporânea. (3a ed., pp.9-36). Petrópolis, RJ: Vozes.

Bzuneck, J. A. (2004b). A motivação do aluno: orientado a metas de realização. In E. Boruchovitch \& J. A. Bzuneck (Orgs.), A motivação do aluno: Contribuições da Psicologia Contemporânea. (3a ed., pp.58-77). Petrópolis, RJ: Vozes.

Darnon, C., Harackiewicz, J. M., Butera, F., Mugny, G., \& Quiamzade, A. (2007). Performance-approach and performance-avoidance goals: When uncertainty makes a difference. Personality and Social Psychology Bulletin, 33, 813827.

Dweck, C. S. (1986). Motivational processes affecting learning. American Psychologist, 41, 1040-1048.

Dweck, C. D., \& Leggett, E. L. (1988). A social-cognitive approach to motivation and personality. Psychological Review, 95(2), 256-273. 
Ee, J., Wang, C. K. J., Koh, C. Tan, O. S. \& Liu, W. C. (2009). Goal orientations and metacognitive skilss of normal technical and normal academic students on project work. Asia Pacific Education Review, 10, 337-344.

Elliot, A. J. \& Church, M. A. (1997). A hierarchical model of approach and avoidance achievement motivation. Journal of Personality and Social Psychology, 72, 218-232.

Givvin, K. B. (2001). Goal orientations of adolescents, coaches, and parents: Is there a convergence of beliefs? Journal of Early Adolescence, 21(2), 228248.

Gottfried, A. E. (1990). Academic intrinsic motivation in elementary and junior high school students. Journal of Educational Psychology, 82(3), 525-538.

Gouveia, V. V., Diniz, P. K. C., Gouveia, R. S. V. \& Cavalcante, J. P. N. (2008). Metas de realização entre estudantes do ensino médio: evidências de validade fatorial e consistência interna de uma medida. Psicologia: Teoria e Pesquisa, 24(4), 535-544.

Guimarães, S. E. R. (2004). Motivação intrínseca, extrínseca e o uso de recompenses em sala de aula. In E. Boruchovitch \& J. A. Bzuneck (Orgs.), A motivação do aluno: contribuições da psicologia contemporânea. (3a ed., pp.37-57). Petrópolis, RJ: Vozes.

Martinelli, S. C., \& Genari, C. H. M. (2009). Relações entre desempenho escolar e orientações motivacionais. Estudos de Psicologia (Natal), 14(1), 13-21.

Middleton, M. J., \& Midgley, C. (1997). Avoiding the demonstration of lack of ability: an uderexplored aspect of goal theory. Journal of Education Psychology, 89(4), 710-728.

Senko, C., \& Harackiewicz, J. M. (2005). Achievement goals, task performance, and interest: Why perceived goal difficulty matters. Personality and Social Psychology Bulletin, 31(12), 1739-1753

Shih, S. S., (2005). Role of achievement goals in children's learning in Taiwan. The Journal of Educational Research, 98(5), 310-318.

Tapia, J. A., \& Montero, I. (2004). Orientação motivacional e estratégias motivadoras na aprendizagem escolar. In C. Coll, A. Marchesi, \& J. Palácios (Orgs.), Desenvolvimento psicológico e educação. (2a ed., pp.177-192). Porto Alegre: Artmed. 
Zenorini, R. P. C., Santos, A. A. A. \& Bueno, J. M. H. (2003). Escala de avaliação das metas de realização: estudo preliminar de validação. Avaliação Psicológica, 2(2), 165-173.

Zenorini, R. P. C. (2007). Estudos para a construção de uma escala de avaliação da motivação para aprendizagem - EMA. Tese de Doutorado, Programa de Pós-Graduação Stricto Sensu em Psicologia, Universidade São Francisco, Itatiba, $138 \mathrm{p}$.

Zenorini, R. P. C. \& Santos, A. A. A. (2009). Escala de Motivação para Aprender (EMAPRE). Relatório Técnico não publicado. Itatiba: Programa de Pósgraduação Stricto Sensu da Universidade São Francisco.

Zenorini, R. da P. C. \& Santos, A. A. A. (2010). Teoria de metas de realização: fundamentos e avaliação. In E. Boruchovitch, J. A. Bzuneck \& S. E. R. Guimarães (Orgs.), Motivação para aprender: aplicações no contexto educativo. (pp.99-125). Petropolis: Vozes.

Contato: rmonteiro@me.com

Recebido em: 08/03/2011

Revisado em: $14 / 05 / 2011$

Aceito em: 04/06/2011 